\title{
Photo-excited zero-resistance states in the GaAs/AlGaAs system
}

\author{
R. G. Mani ${ }^{1, *}$ \\ ${ }^{1}$ Harvard University, Gordon McKay Laboratory of Applied Science, 9 Oxford Street, Cambridge, MA 02138, USA
}

(Dated: November 7, 2018)

\begin{abstract}
The microwave-excited high mobility two-dimensional electron system exhibits, at liquid helium temperatures, vanishing resistance in the vicinity of $B=[4 /(4 j+1)] B_{f}$, where $B_{f}=2 \pi f m^{*} / e, \mathrm{~m}^{*}$ is an effective mass, e is the charge, and $f$ is the microwave frequency. Here, we summarize some experimental results.

Journal-Reference: International Journal of Modern Physics B 18, 3473 (2004)
\end{abstract}

\section{INTRODUCTION}

The creation, detection, and study of exciton condensates in semiconductors constitute interesting problems in condensed matter physics.[1] Exciton populations are usually created by photo-exciting a semiconductor, so that the electron-hole pairs consist of conduction band electrons and valence band holes. Then, optical techniques are employed to detect and study the excitonic system.[1, 2] Recently, there has also been interest in the possibility of electrical detection, and the realization without photoexcitation, of such exotic states in a bilayer quantum Hall system, where the excitons result from the pairing of electrons in one quantum Hall layer with holes in the neighboring layer.[1, 3, 4] This report examines a hybrid scenario where photoexcitation serves to create inter-Landau level, instead of inter-band, excitations in a quantum Hall system, as electrical detection is employed to detect the system response.[5, 6, 7, 8, 9, 10, 11, 12, 13] In particular, we study transport in a semiconductor single-layer two-dimensional electron system, where $m i$ crowave photo-excitation produces a steady state density of inter-Landau level excitations ("excitons"). Surprisingly, such a system exhibits novel zero-resistance states without concomitant Hall quantization, at low temperatures, $T$, about magnetic fields, $B=(4 / 5) B_{f}$ and $B=$ $(4 / 9) B_{f}$, where $B_{f}=2 \pi f m^{*} / e, m^{*}$ is an effective mass, $e$ is the electron charge, and $f$ is the radiation frequency. [9] The experimental observations have led to a broad theoretical study of this situation.[14, 15, 16, 17, 18, 19, 20, 21, 22, 23, 24, 25, 26, 27, 28, 29, 30, 31, 32, 33, 34, 35]

At the present, it is uncertain whether the observed zero-resistance states should be viewed as a novel condensate with a role for excitons, although we had suggested this possibility. [9, 32] Theoretical studies have realized radiation-induced magneto-resistivity oscillations through various approaches.[14, 16, 18, 22, 23, 24, 28, 29, 30, 31] The observed zero-resistance states have then been attributed to a negative resistivity instability, and current-domain formation.[17] As there remain many open questions from both the experimental and theoretical perspectives, we examine here just the experimental situation, and refer the reader to the literature for further theory.

\section{EXPERIMENT}

Experiments were carried out on standard devices fabricated from GaAs/AlGaAs heterostructures. The best material was typically characterized by an electron density, $n(4.2 \mathrm{~K}) \approx 3 \times 10^{11} \mathrm{~cm}^{-2}$, and an electron mobility, $\mu$, up to $1.5 \times 10^{7} \mathrm{~cm}^{2} / \mathrm{Vs}$. Standard four-terminal lockin based electrical measurements were performed with
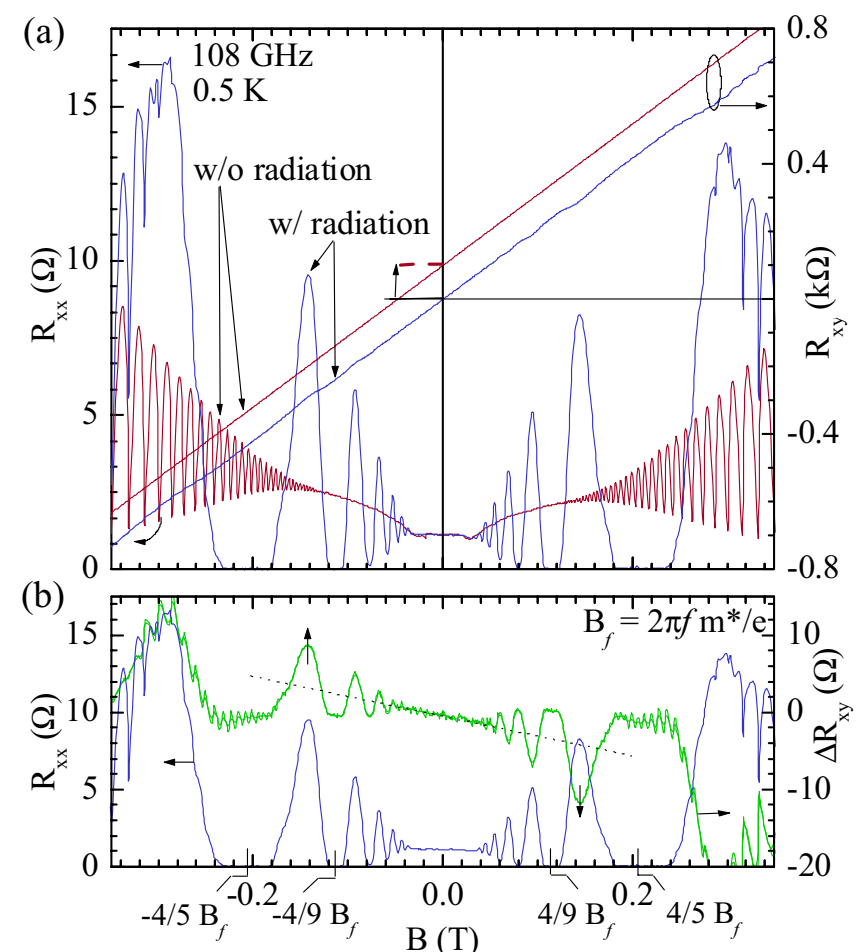

FIG. 1: (a): Transport measurements with (w/) and without (w/o) microwave radiation at $f=108 \mathrm{GHz}$. Radiation induced vanishing $R_{x x}$ is observable about $(4 / 5) B_{f}$ and $(4 / 9) B_{f}$. A comparison of the w/ and w/o radiation $R_{x y}$ indicates antisymmetric-in-B oscillations in $R_{x y}$ under photoexcitation, which correlate with the $R_{x x}$ oscillations. Here, the $\mathrm{w} / \mathrm{o}$ radiation Hall data have been offset for the sake of clarity. (b): The radiation induced change in the Hall resistance, $\Delta R_{x y}$, is shown along with $R_{x x}$. Note that the antisymmetricin-B $\Delta R_{x y}$ oscillations. 


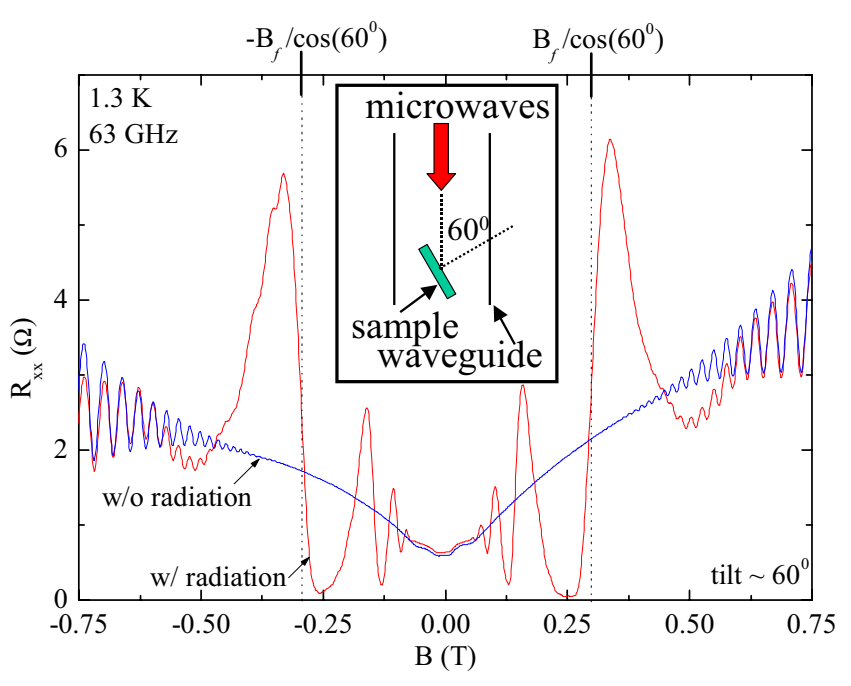

FIG. 2: The effect of microwave radiation on a tilted 2DES, when the tilt angle is $60^{\circ}$, see inset. Here, the abscissa shows the applied (total) magnetic field. Typically, the radiationinduced magnetoresistance oscillations are mostly sensitive to the normal component of $B$. Thus, the characteristic $B$-scale under tilt is given by $B_{f} / \cos (\theta)$, where $\theta$ is the tilt angle.

the sample mounted inside a waveguide and immersed in pumped liquid He-3 or He-4. Electromagnetic (EM) waves in the microwave part of the spectrum were generated using tunable sources. The EM waves were directed to the sample through the waveguide. Further details appear elsewhere.[9]

The data of Fig. 1(a) illustrate the radiation-induced effect on the diagonal $\left(R_{x x}\right)$ and Hall resistances $\left(R_{x y}\right)$, when the specimen is excited with microwaves at $f=108$ GHz. In Fig. 1(a), the application of microwaves induces giant oscillations in $R_{x x}$, which are characterized by the property that the $R_{x x}$ under photoexcitation drops below the dark $R_{x x}$ over wide $B$ intervals. Thus, at approximately $B_{f} / B=j$ and $B_{f} / B=j+1 / 2$, with $j=1,2,3 \ldots$ , the $R_{x x}$ under photoexcitation is equal to the dark $R_{x x}$. For $j<B_{f} / B<j+1 / 2$, the photo-excited $R_{x x}$ is reduced with respect to the dark $R_{x x}$. On the other hand, for $j+1 / 2<B_{f} / B<j+1$, the irradiated $R_{x x}$ is enhanced with respect to the dark $R_{x x}$. A remarkable feature in the data are the zero-resistance states which are manifested about $B_{f} / B=j+1 / 4$, i.e., in the vicinity of $B=(4 / 5) B_{f}$ and $B=(4 / 9) B_{f}$. These $R_{x x}$ minima exhibit activated transport vs. the temperature, which is suggestive, from the experimental perspective, of a possible radiation-induced gap in the electronic spectrum.[9]

The data of Fig. 1a also illustrate the influence of radiation on the Hall effect. Here, a comparison of the dark $R_{x y}$ with the photo-excited $R_{x y}$ reveals subtle microwave induced oscillations, which are period commensurate with the radiation induced $R_{x x}$ oscillations. [9, 12] Yet,

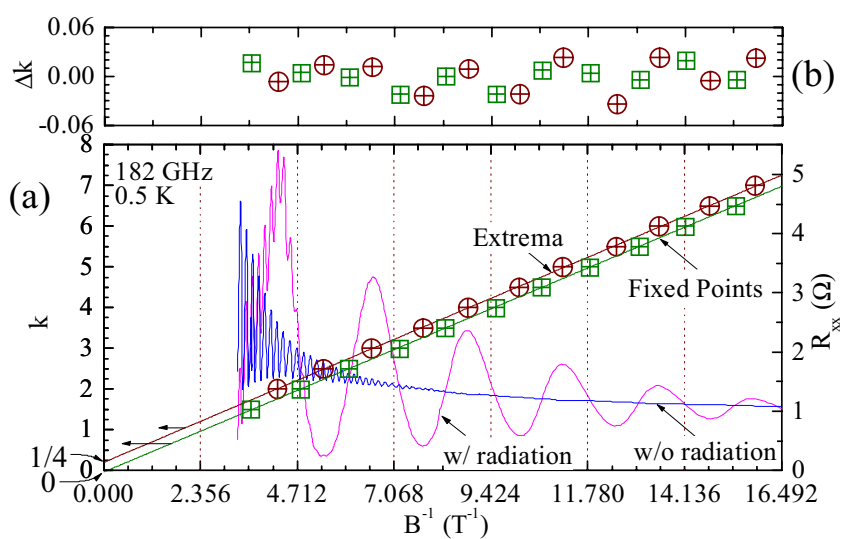

FIG. 3: (a) Right ordinate: $R_{x x}$ with (w/) and without (w/o) radiation at $182 \mathrm{GHz}$ is plotted vs. $B^{-1}$. Left ordinate: Halfcycle plots of the extremal- and fixed- points in the radiation induced resistance oscillations. The slope of the linear fit is proportional to $m^{*} / m$, while the ordinate intercepts measure the phase, which are " $1 / 4$ " and " 0 " for the extremal and fixed points, respectively. (b): The residue in the labels, $\Delta k=$ $k-k_{F I T}$, is shown as a function of $B^{-1}$.

there do not occur microwave-induced plateaus in the Hall effect that coincide with the radiation-induced vanishing $R_{x x}$ in the vicinity of $\mathrm{B}=(4 / 5) B_{f}$ and $(4 / 9) B_{f}$. In order to highlight these radiation-induced changes in $R_{x y}, \Delta R_{x y}=R_{x y}^{\text {excited }}-R_{x y}^{\text {dark }}$ is shown along with $R_{x x}$ in Fig. 1(b). A comparison of Fig. 1(a) and Fig. 1(b) shows clearly that $R_{x y}$ is reduced in magnitude over the B-intervals, where $R_{x x}$ is enhanced by the radiation. On the other hand, as the diagonal resistance vanishes upon microwave excitation, as in the vicinity of $B=(4 / 5) B_{f}$, for example, the correction $\Delta R_{x y}$ also vanishes.

As the period of the radiation induced oscillations (see Fig. 1) are mostly sensitive to the $B$-component that is perpendicular to the plane of the 2DES, they tend to span a wider $B$-interval, at a finite tilt angle of the specimen. Thus, under tilt, the characteristic field scale becomes $B_{f} / \cos (\theta)$, and the dark $R_{x x}$ intercepts the photoexcited $R_{x x}$ in the vicinity of $B=\left[B_{f} / \cos (\theta)\right] / j$ and $B=\left[B_{f} / \cos (\theta)\right] /(j+1 / 2)$, with $j=1,2,3 \ldots$ These features can be detected in Fig. 2

The phase of the radiation induced $R_{x x}$ oscillations is an important parameter because it can be used to compare experiment to theory.[9, 10, 14, 16, 18] To determine this observable, $R_{x x}$ maxima and minima of $182 \mathrm{GHz}$ data (see Fig. 3) are initially labelled by integers and half-integers, respectively, assuming a cosine waveform. The extrema labels, denoted by the index $k$ in Fig. 3, are then plotted vs. extremal values of $B^{-1}$, and a linear fit, $k_{F I T}$, is applied to determine the slope, $d k_{F I T} / d B^{-1}$ $=B_{f}$, and the ordinate intercept. In Fig. 3, the ordinate intercept for the fit of extremal data points at $1 / 4$ demonstrates a 1/4-cycle phase shift of the extrema with 


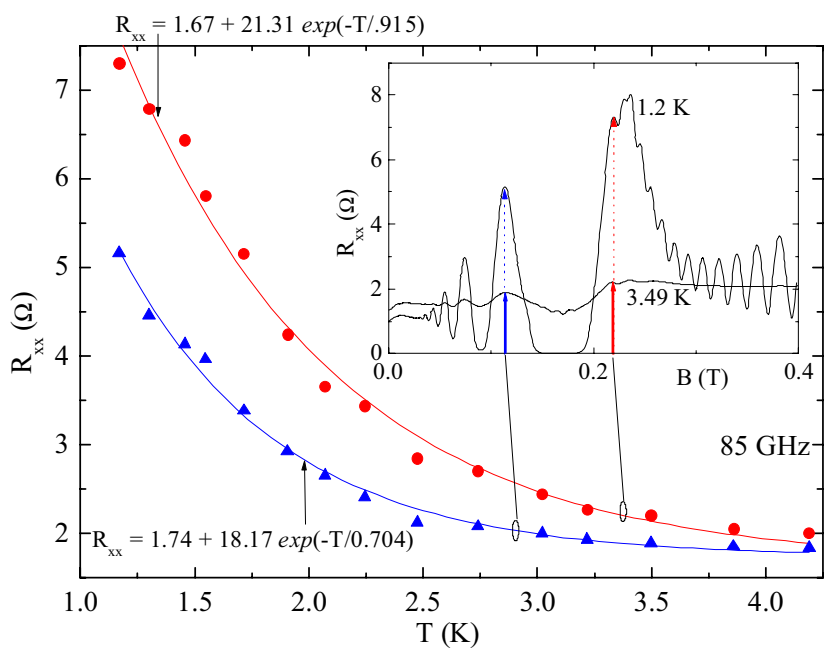

FIG. 4: (a) Inset: The diagonal resistance, $R_{x x}$, is exhibited vs. the magnetic field, $B$, with microwave excitation at 85 GHz. The main panel exhibits the temperature dependence of the first and second resistance maxima, along with empirical fits to the data.

respect to the assumed (cosine) waveform.[9] Thus, the plot (Fig. 3(a)) demonstrates that the phase is broadly consistent with a lineshape $R_{x x} \approx-\sin \left(B_{f} / B\right)$, i.e., resistance minima about $h f / \hbar \omega_{c}=j+1 / 4$, and resistance maxima about $h f / \hbar \omega_{c}=j+3 / 4$. A similar analysis of the nodes or fixed points suggests that they are characterized by $h f / \hbar \omega_{c}=j$, or $h f / \hbar \omega_{c}=j+1 / 2$, see Fig. 3 .

In prior work, we have shown that the radiation induced resistance minima exhibit activated transport, i.e., $R_{x x} \propto \exp \left(-T_{0} / T\right) \cdot[9]$ As a supplement to that previous report, we examine the $T$-dependence of the resistance maxima in Fig. 4. Thus, the inset in Fig. 4 shows $R_{x x}$ under microwave excitation, with a pair of marked maxima, whose $T$-evolution is followed in the main part of the figure. Fig. 4 shows that the $R_{x x}$ maxima increase rapidly with decreasing temperature, and the variation can be empirically represented by an exponential function. The $T$-variation of the maxima have been attributed by Dmitriev et al., to the $T$-dependence of the inelastic scattering rate.[31]

Measurements examining possible reflection/emission and transmission characteristics of the high mobility 2DES under microwave excitation are illustrated in Fig. 5 and Fig. 6. For these experiments [see inset, Figs. 5 and 6$]$, a resistance sensor was placed immediately above or below the sample.[9]

Fig. 5 exhibits the dark, and irradiated at $30 \mathrm{GHz}$, $R_{x x}$, along with the sensor $R_{S 1}$ response under photoexcitation. The irradiated $R_{x x}$ shows giant microwaveinduced oscillations. Note that, in this low- $f$ case, the irradiated $R_{x x}$ crosses the dark $R_{x x}$ also near $2 B_{f}$. Also

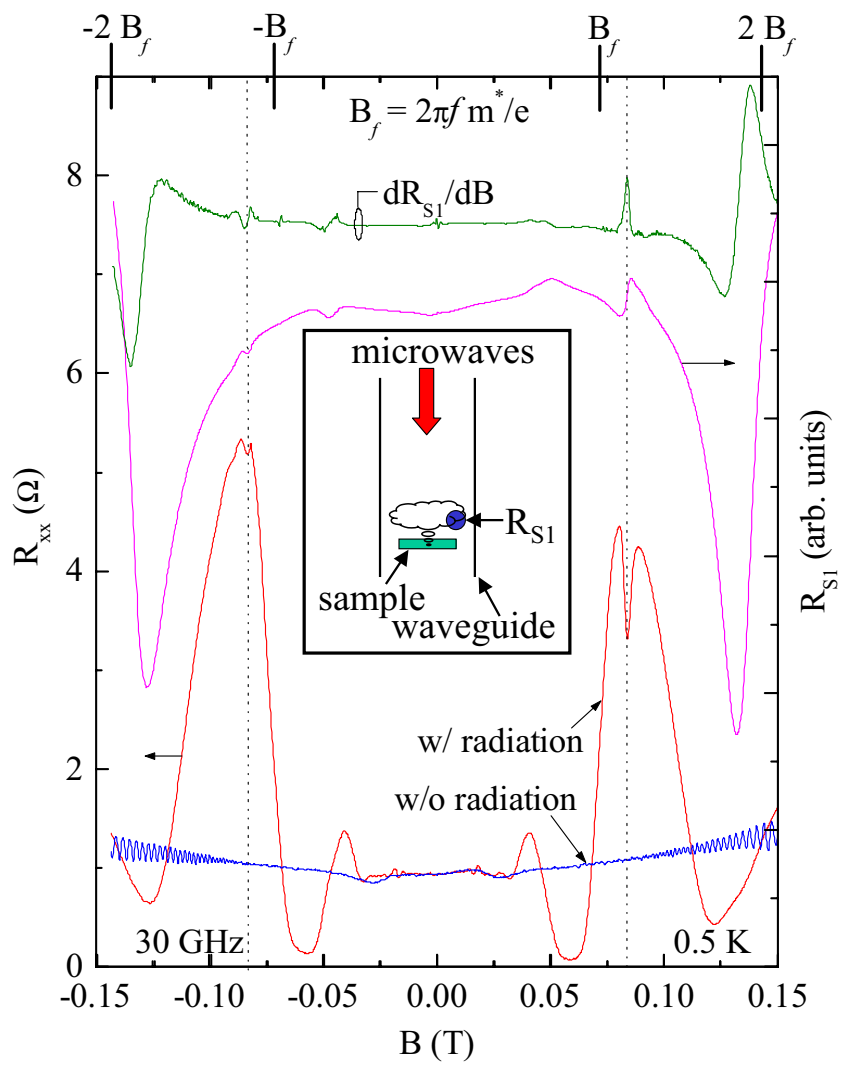

FIG. 5: This figure examines the reflection/emission characteristics of the irradiated 2DES. Thus, the figure exhibits the dark, and the $30 \mathrm{GHz}$ photo-excited $R_{x x}$, as well as the sensor $R_{S 1}$ response under photoexcitation. Also shown is the numerical derivative, $d R_{S 1} / d B$. The $R_{S 1}$ signal shows a pair of significant features between $B_{f}$ and $2 B_{f}$, as the near $2 B_{f}$ feature coincides with a second harmonic, two-photon process. The inset illustrates the measurement configuration.

shown in the figure is $d R_{S 1} / d B$, which serves to bring out weak features in the $R_{S 1}$. The $R_{S 1}$ signal shows a pair of significant features between $B_{f}$ and $2 B_{f}$. Here, the largest feature might originate from a two-photon process. In the derivative signal, there is a very weak feature near - $B_{f}$ (but not at $+B_{f}$ ), which could signify electrically detected cyclotron resonance. The origin of other structure in the sensor signal is not fully understood.

Some transmission measurements are shown in Fig. 6 . Here, the optimal radiation induced $R_{x x}$ response was observed in the vicinity of $-9 \mathrm{~dB}$. A further increase of the radiation intensity $(\mathrm{dB} \rightarrow 0)$ produced a reduction in the peak height along with an increase in the resistance at the minima (see Fig. 6(a)). At the same time, the transmission sensor (see Fig. 6(b)) indicated structure at $B>B_{f}$, which correlated with a strong radiationinduced decrease in $R_{x x}$ just above 0.3 Tesla. Observed oscillations in $R_{x x}$ below $B_{f}$ (Fig. 6(a)) were imperceptible, however, in the detector response (see Fig. 6(b)). 


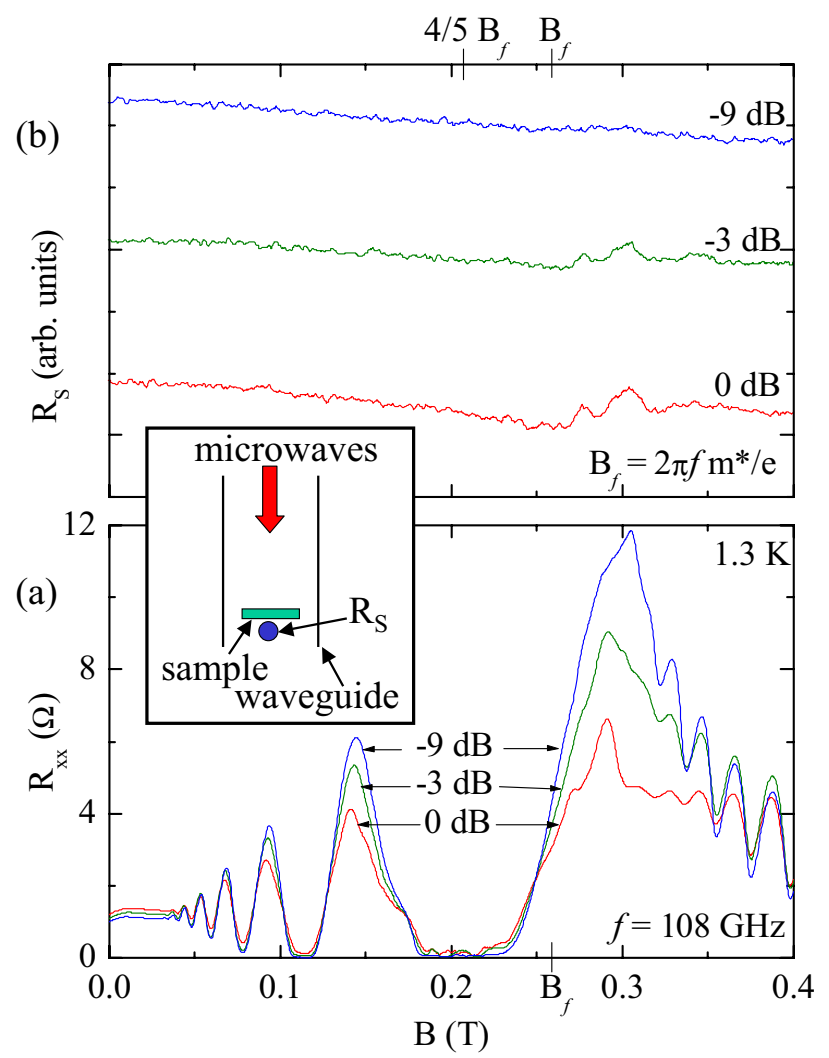

FIG. 6: The figure illustrates the transmission characteristics of the 2DES under irradiation. As shown in the inset, a resistance sensor below the sample serves as the radiation detector. $R_{x x}$ is shown in (a) while the detector resistance, $R_{S}$, is shown in (b). The bottom panel (a) shows that, when the power attenuation factor exceeds $-9 \mathrm{~dB}$, the oscillation amplitude decreases with increasing radiation intensity. At the same time, the detector response (b) suggests non monotonic transmission above $B_{f}$. Oscillations in $R_{x x}$ below $B_{f}$ appear imperceptible in $R_{S}$.

It is also interesting to examine the effect of simultaneous excitation of the specimen at two different microwave frequencies.[15, 24] Thus, Fig. 7 exhibits measurements with excitation of the specimen at $15 \mathrm{GHz}$ only, and at $50 \mathrm{GHz}$ only. Here, at $50 \mathrm{GHz}$, there occur a number of radiation-induced oscillations and one broad zeroresistance state about $(4 / 5) B_{f}$. On the other hand, at $15 \mathrm{GHz}$, the radiation seems to produce a non-monotonic variation in $R_{x x}$, mostly above $B_{f}$. Simultaneous excitation at two-frequencies produces a $R_{x x}$ trace that looks very much like the trace at the higher $f$, i.e., $50 \mathrm{GHz}$. An interesting feature is observed here by comparing the $f_{1}$ AND $f_{2}$ signals at $B \approx 0.07 \mathrm{~T}$ and $B \approx 0.14 \mathrm{~T}$. At both these $B$, the peak amplitude of the $f_{1}$ AND $f_{2}$ signal is reduced with respect to the $f_{2}$ signal. Yet, one observes that in the $B \approx 0.07 \mathrm{~T}$ case, only $f_{1}$ produces an enhancement, while in the $B \approx 0.14 \mathrm{~T}$ case, it produces a resistance suppression, with respect to the dark signal.

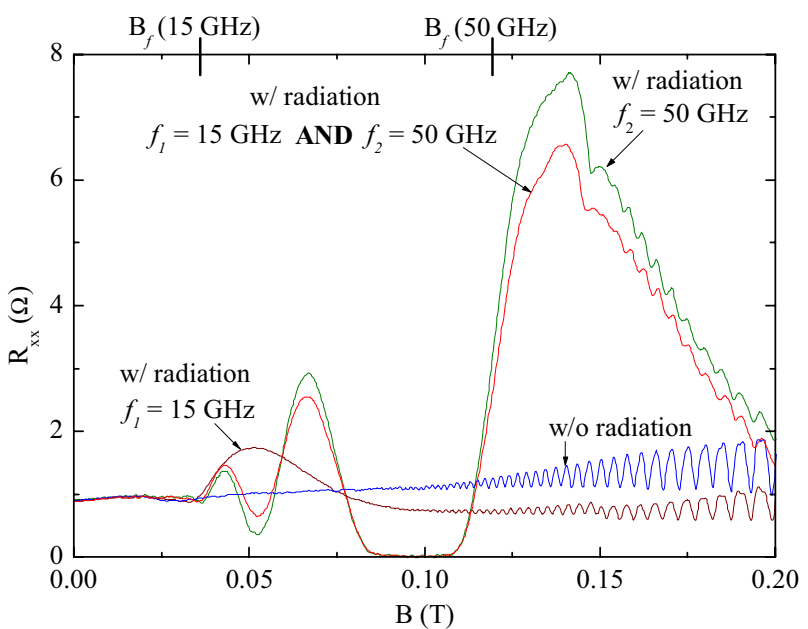

FIG. 7: This figure traces the effect of simultaneously exciting the GaAs/AlGaAs device at two microwave frequencies. Thus, we have exhibited the dark (w/o radiation) magnetoresistance, the $R_{x x}$ obtained under excitation at $50 \mathrm{GHz}$ only, the $R_{x x}$ with excitation at $15 \mathrm{GHz}$ only, and the $R_{x x}$ obtained under simultaneous excitation at $15 \mathrm{GHz}$ and $50 \mathrm{GHz}$. The latter looks mostly like the trace obtained at $50 \mathrm{GHz}$. The characteristic $B_{f}$ have been marked the figure.

Thus, perhaps, one should attribute the small decrease in the amplitude of the $f_{1}$ AND $f_{2}$ signal to a "breakdown" type heating effect, due to the increased radiation intensity resulting from the combined excitation.

\section{SUMMARY}

In summary, radiation induced zero-resistance states and associated oscillations in the high mobility 2DES exhibit a rich phenomenology. Here, we have shown that (a) $R_{x x}$ exhibits saturation at zero-resistance under microwave excitation in the vicinity of $[4 /(4 j+1)] B_{f}$, with concomitant vanishing Hall resistance correction, $\Delta R_{x y}$, see Fig. 1, (b) the period of the radiation induced oscillations are mostly sensitive of the normal component of the magnetic field, see Fig. 2, (c) the oscillatory minima are shifted by $1 / 4$ cycle with respect to the $h f / \hbar \omega_{c}=j$ condition, see Fig. 3, (d) the oscillatory resistance maxima increase strongly with decreasing temperature, see Fig. 4, (e) a response is observable in the transmission and reflection signals, mostly above $B_{f}$, see Figs. 5 and 6 , and (e) simultaneous photoexcitation at two radiation frequencies seems not to produce simple superposition, see Fig. 7. 


\section{Acknowledgements}

We acknowledge discussions with $\mathrm{K}$. von Klitzing, V. Narayanamurti, J. H. Smet, and W. B. Johnson, and the receipt of high quality MBE material from V. Umansky.

* Electronic address: mani@deas.harvard.edu

[1] B. G. Levi, Physics Today 57 (7), 21 (2004).

[2] L. V. Butov et al., Nature 418, 751 (2002); Phys. Rev. Lett. 92, 117404 (2004).

[3] I. B. Spielman et al., Phys. Rev. Lett. 84, 5808 (2000); M. Kellogg et al., Phys. Rev. Lett. 88, 126804 (2002).

[4] R. E. Prange and S. M. Girvin, (eds) The Quantum Hall Effect, 2nd Ed., New York: Springer-Verlag, 1990.

[5] R. Fitzgerald, Phys. Today 56 (4), 24-27 (2003).

[6] R. G. Mani et al., Bull. Am. Phys. Soc. 46, p. 972 (2001).

[7] M. Zudov, R. Du, J. Simmons, and J. Reno, Phys. Rev. $B$ 64, 201311 (2001).

[8] P. D. Ye et al., Appl. Phys. Lett. 79, 2193 (2001).

[9] R. G. Mani et al., Nature (London) 420, 646 (2002); Phys. Rev. B 69, 193304 (2004); cond-mat/0305507; cond-mat/0306388; Phys. Rev. B 69, 161306 (2004); Phys. Rev. Lett. 92, 146801 (2004); R. G. Mani, Physica E 22, 1 (2004); cond-mat/0407143.

[10] M. Zudov et al., Phys. Rev. Lett. 90, 046807 (2003); C. L. Yang et al., Phys Rev. Lett. 91, 096803 (2003); R. R. Du et al., Physica E 22, 7 (2004).

[11] S. I. Dorozhkin, JETP Lett. 77, 577 (2003).

[12] S. A. Studenikin et al., Sol. St. Comm. 129, 341 (2004)

[13] A. E. Kovalev et al., Sol. St. Comm. 130, 379 (2004).

[14] V. I. Ryzhii, Sov. Phys. - Sol. St. 11, 2078-2080 (1970).
[15] J. C. Phillips, Sol. St. Comm. 127, 233 (2003).

[16] A. Durst et al., Phys. Rev. Lett. 91, 086803 (2003); A. C. Durst and S. M. Girvin, Science 304, 1752 (2004).

[17] A. V. Andreev, I. L. Aleiner, and A. J. Millis, Phys. Rev. Lett. 91, 056803 (2003).

[18] J. Shi and X. C. Xie, Phys. Rev. Lett. 91, 086801 (2003).

[19] A. A. Koulakov and M. E. Raikh, Phys. Rev. B 68, 115324 (2003).

[20] F. S. Bergeret, B. Huckestein, and A. F. Volkov, Phys. Rev. B 67, 241303 (2003).

[21] I. A. Dmitriev, A. D. Mirlin, and D. G. Polyakov, Phys. Rev. Lett. 91, 226802 (2003).

[22] X. L. Lei and S. Y. Liu, Phys. Rev. Lett. 91, 226805 (2003).

[23] V. Ryzhii and V. Vyurkov, Phys. Rev. B 68, 165406 (2003).

[24] D. H. Lee and J. M. Leinaas, Phys. Rev. B 69, 115336 (2004).

[25] V. Ryzhii, Phys. Rev. B 68, 193402 (2003).

[26] M. G. Vavilov and I. L. Aleiner, Phys. Rev. B 69, 035303 (2004).

[27] A. F. Volkov and V. V. Pavlovskii, Phys. Rev. B 69, 125305 (2004).

[28] V. Ryzhii and A. Satou, J. Phys. Soc. Jpn. 72, 2718 (2003).

[29] K. Park, Phys. Rev. B 69, 201301 (2004).

[30] V. Ryzhii, R. Suris, and B. Shchamkhalova Physica E 22, 13 (2004).

[31] I. A. Dmitriev et al., cond-mat/0310668.

[32] S. Fujita and K. Ito, cond-mat/0402174.

[33] M. Vavilov et al., cond-mat/0405377.

[34] C. Joas, M. E. Raikh, and F. von Oppen, condmat/0405443.

[35] A. E. Patrakov and I. L. Lyapilin, cond-mat/0405517. 